\title{
Electrode positioning to investigate the changes of the thoracic bioimpedance caused by aortic dissection - a simulation study
}

\author{
V. Badeli ${ }^{1,3}$, G. M. Melito ${ }^{2}$, A. Reinbacher-Köstinger ${ }^{1}, \mathrm{O}$ Bíró $^{1}$, and K. Ellermann ${ }^{2}$ \\ 1. Institute of Fundamentals and Theory in Electrical Engineering, Graz University of Technology, Graz, Austria \\ 2. Institute of Mechanics, Graz University of Technology, Graz, Austria \\ 3. E-mail any correspondence to: Vahid.badeli@tugraz.at
}

\begin{abstract}
Impedance cardiography (ICG) is a non-invasive method to evaluate several cardiodynamic parameters by measuring the cardiacsynchronous changes in the dynamic transthoracic electrical impedance. ICG allows us to identify and quantify conductivity changes inside the thorax by measuring the impedance on the thorax during a cardiac cycle. Pathologic changes in the aorta, like aortic dissection, will alter the aortic shape as well as the blood flow and consequently, the impedance cardiogram. This fact distorts the evaluated cardiodynamic parameters, but it could lead to the possibility to identify aortic pathology. A 3D numerical simulation model is used to compute the impedance changes on the thorax surface in case of the type B aortic dissection. A sensitivity analysis is applied using this simulation model to investigate the suitability of different electrode configurations considering several patientspecific cases. Results show that the remarkable pathological changes in the aorta caused by aortic dissection alters the impedance cardiogram significantly.
\end{abstract}

Keywords: Aortic dissection; impedance cardiography; numerical simulation; sensitivity analysis
The false lumen represents the blood-filled space between the dissected layers of the aortic wall, while the true lumen is the usual passageway of blood.

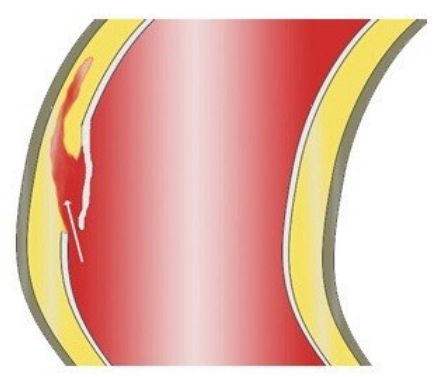

a.

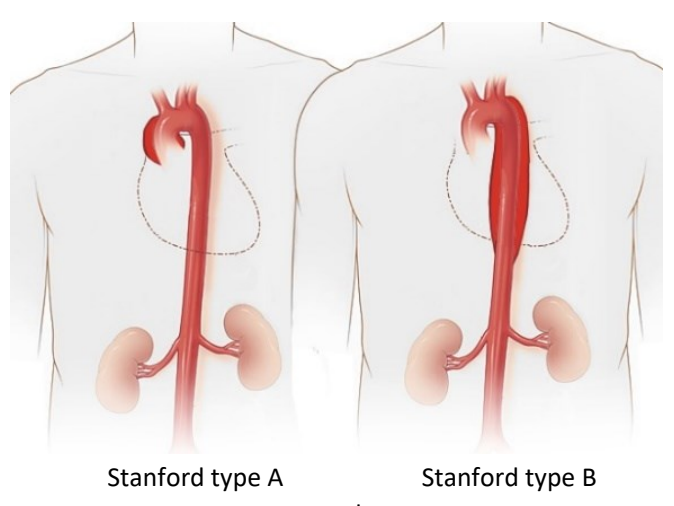

b.

Fig.1: a) Intimal tear in the aorta [2]. b) Aortic dissection types (Stanford system) [3]. 
The ADs are classified as Stanford type A (proximal) or B (distal) in the Stanford system (figure 1.b). If the ascending aorta is involved (Stanford type A), an acute condition with a high mortality rate within a few hours occurs in most cases due to the high blood pressure right after the aortic heart valve. On the other hand, Stanford type B cases (in the descending aorta) may become chronic, which means that the onset of the dissection dates back more than 14 days and patients can often be treated with medical therapy. In both cases, the symptoms of AD patients are sudden severe chest or upper back pain, which are not easily assignable to this disease.

Detecting an AD can be difficult because the symptoms are similar to those of a variety of health problems. Ultrasound Scanning (sonography), Magnetic Resonance Imaging (MRI) and Computerized Tomography (CT) are expensive techniques currently used for this purpose, with experts needed to read and interpret the images. Nevertheless, an easy to use and still reliable method for preidentification of $A D$ would be beneficial. Furthermore, tracking the development of the disease, such as false lumen expansion and false lumen thrombosis can be very helpful for the medical management of AD.

The presence of a false lumen alters the aortic haemodynamics and also changes the tissue distribution in the thorax. These changes can be identified and quantified by bioimpedance techniques such as impedance cardiography (ICG). In ICG, a current field longitudinally across a segment of the thorax is applied using a constant low magnitude and high-frequency alternating current. It is a non-invasive, safe, easy to use and low-cost method for measuring several cardiodynamic parameters (e.g. the Stroke Volume (SV) and the Cardiac Output (CO)) continuously [4]. Besides, this method is portable, and the analysis could be automated.

By injecting a low-amplitude alternating current into the thorax and measuring the voltage drop $\Delta V$ on the thorax, impedance changes can be evaluated during a cardiac cycle. The negative of the first time-derivative of the impedance signal $-|d \underline{Z} / d t|$ is known as the impedance cardiogram. Since the conductivity of the blood-filled aorta is much higher than that of the surrounding tissue types, changes of the measured impedance are strongly related to changes in the aorta. Since both the blood volume and the blood flow will change in the case of an $A D$, an altered impedance cardiogram can be expected, which makes the ICG a good candidate for diagnosis and monitoring purposes [5-7].

A 3D numerical simulation model is used to compute the impedance changes on the thorax surface in case of the type $B$ aortic dissection. A sensitivity analysis through the Global Sensitivity Analysis (GSA) technique is applied to investigate different electrode configurations in the simulation model with different input parameters to cover as many patientspecific cases as the dimension of the input space. The final aim would be finding the desired electrode configuration, which gives the highest difference between the impedance cardiograms of the healthy condition and the ones with the AD.

\section{Methods}

Sources of impedance changes during a cardiac cycle The measured electrical impedance without respiratory or cardiac activity is known as static thoracic base impedance $Z_{0}$. Upon ventricular ejection, a time-dependent pulsatile impedance change $\Delta \underline{Z}(t)$ is obtained. When $\Delta \underline{Z}(t)$ is superimposed on $Z_{0}$, the time variable total transthoracic impedance $\underline{Z}(t)$ is registered.

By eliminating the oscillating cardiac-asynchronous respiratory component, $\underline{Z}(t)$ comprises a static DC component $Z_{0}(22 \Omega-45 \Omega)$ and a dynamic AC component $\Delta \underline{Z}(t)(0.1 \Omega-0.2 \Omega)$ which is synchronous to cardiac activity $[8,9]$. In many studies, sources of the thoracic impedance changes have been investigated, and a consensus is lacking in the origins of cardio-synchronous impedance changes due to different model assumptions. Hereof different approaches are listed in [10]. Of course, simulation of transthoracic bioimpedance signals considering all possible timedependent sources is impossible. Also, comparing experimental results obtained from dissected patients with earlier measurements in healthy states, is practically infeasible. Nevertheless, since the discrepancy between the healthy and the dissected state and not the evaluation of absolute measurement values is in the focus of this work, only the velocity induced blood conductivity variation and the volumetric changes of the aorta are considered as the sources of $\Delta \underline{Z}(t)$ in a healthy case. It should be noted that the magnitude of $Z_{0}$ not only varies among individuals and the frequency of the applied current but also with the electrode configuration used for signal acquisition.

\section{Volumetric changes of the blood-filled aorta}

The volumetric expansion of the blood-filled aorta changes corresponding to the cardiac pulse wave. For the sake of simplicity, a spatial average time-dependent cross-sectional radius of the aorta has been used in the simulation model for two sections separately, the aortic arch and the descending aorta, see figure 2 . The data are based on measurements provided in [11] from a young, healthy male volunteer at rest.

\section{Velocity induced blood conductivity variation}

The electrical properties of resting blood mainly depend on the volume fraction of red blood cells (RBCs) called haematocrit, the temperature, and cell shape. However, the electrical properties of flowing blood are found to be influenced by the flow rate [12]. 


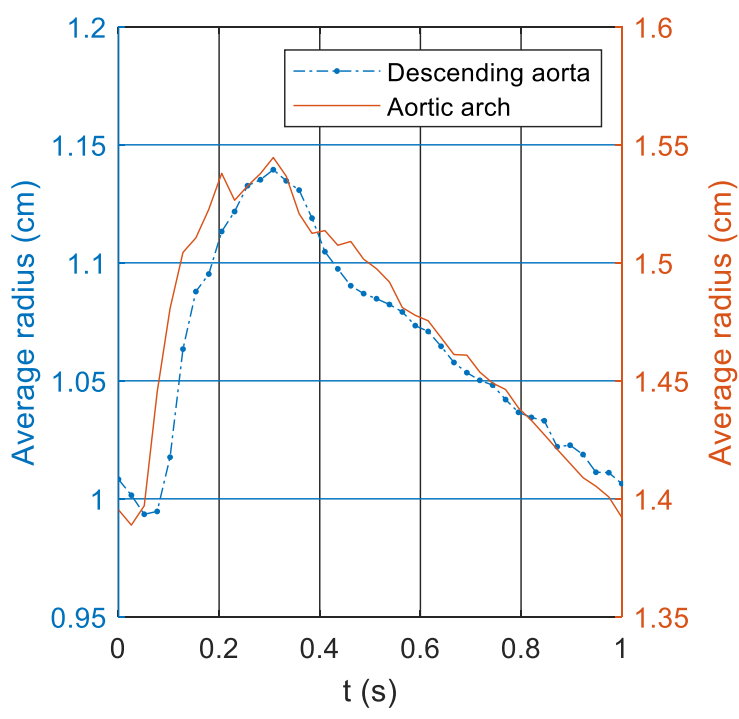

Fig.2: The spatial average time-dependent cross-sectional radius of the aortic arch and the descending aorta during one cardiac cycle.

A spatial average time-dependent velocity of the blood flowing inside the aorta, taken from the experimental data provided in [11], has been taken into account for the aortic arch and the descending aorta (figure 3). During the systolic phase of a cardiac cycle, the heart contracts to pump blood

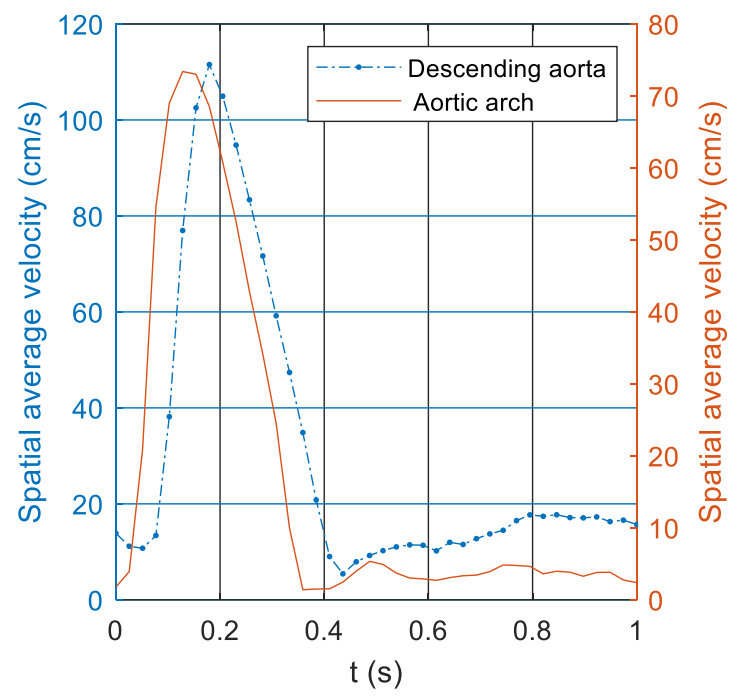

Fig.3: The spatial average time-dependent blood velocity in the aortic arch and the descending aorta.

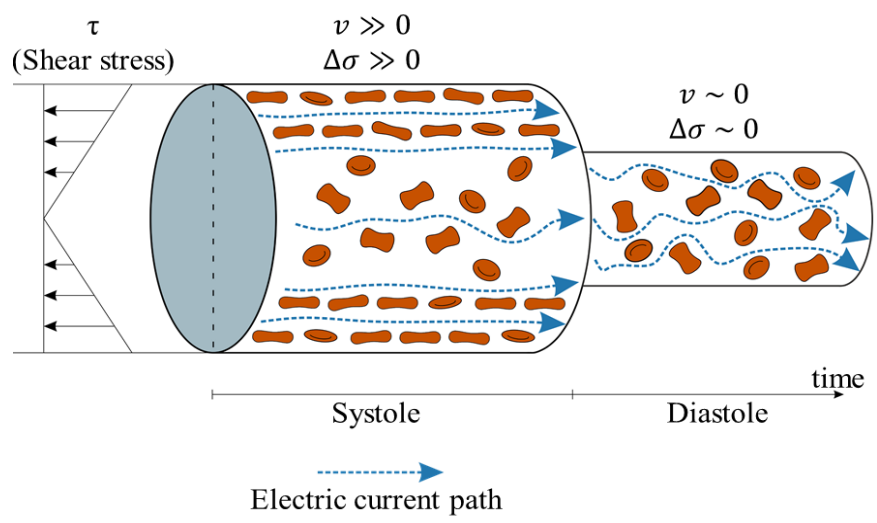

Fig.4: Orientation and deformation of RBCs in a blood vessel during the systole and diastole. into the aorta, and in the diastolic phase, the heart relaxes after contraction. This pulsatile blood flow causes the variation of blood conductivity inside the aorta. The reason is the orientation and deformation of the RBCs in case of flowing blood. At higher velocities, the shear stress increases, which consequently deforms the RBCs in the layer with the highest stress close to the vessel wall and also aligns them throughout the vessel. Both effects lead to a higher conductivity than the resting blood (figure 4) $[13,14]$.

The Maxwell-Fricke equation for the conductivity of blood reads [13]:

$$
\frac{\sigma_{\text {blood }}}{\sigma_{\text {plasma }}}=\frac{1-H}{1+(C-1) H}
$$

where $\sigma_{\text {blood }}$ and $\sigma_{\text {plasma }}$ are the conductivities of blood and plasma, respectively, $H$ is the haematocrit expressed as the volume fraction of RBCs relative to the total blood volume, and $C$ is a factor that depends on the geometry and orientation of the RBCs. Based on the formulation described in [13] (blood is considered as a Newtonian fluid with a steady flow), the blood conductivity changes as a function of reduced average velocity $\langle v / R\rangle(v$ is the spatial average blood velocity, and $R$ is the spatial average cross-sectional radius of the aorta), for different haematocrit levels are shown in figure 5. From this figure, it is evident that more conductivity changes of blood exist with higher haematocrit levels. It is also evident from this figure that the conductivity changes mostly occur in the lower blood velocities and for higher velocities, the slope of the $\Delta \sigma$ curve decreases significantly.

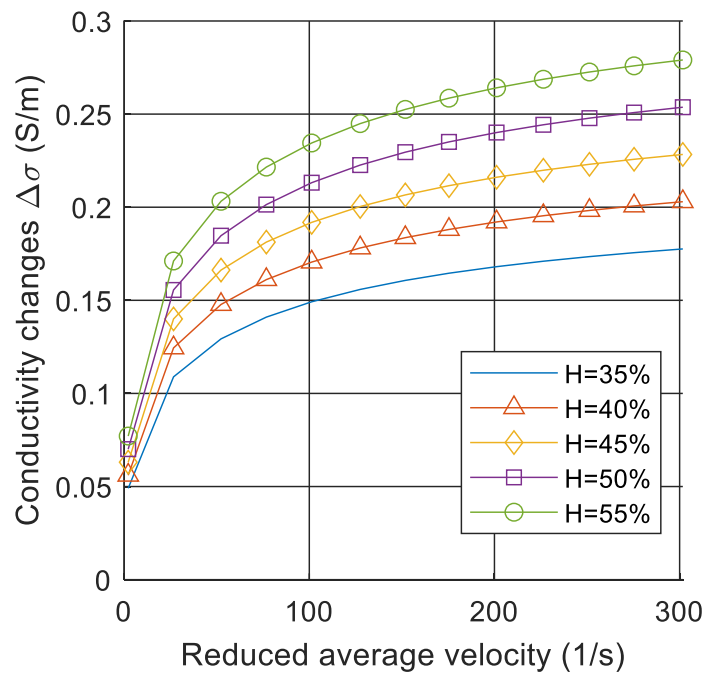

Fig.5: The blood conductivity changes as a function of reduced average velocity $\langle v / R\rangle$ for different haematocrit $(H)$ levels.

In $[15,16]$, it is shown that the blood conductivity during pulsatile blood flow is not the same at any given velocity during acceleration and deceleration. This disparity is a consequence of the RBCs inability to achieve complete randomization at end-systole, which leads to less but still 
considerable conductivity changes during the cardiac cycle. However, for simplicity, conductivity changes which are shown in figure 5 have been assumed in the simulation model.

\section{Simulation model}

Geometry, physics and formulation

A 3D numerical simulation model is used to investigate the changes in the electric potential and the impedance changes on the thorax surface. The model has been set up in COMSOL Multiphysics [17] for the underlying time-harmonic current flow problem. Since the duration of the cardiac cycle is much higher than the period of the injecting current, simulations can be performed in the frequency domain. The electric potential drop is evaluated between the measuring electrodes by solving the Laplace equation for the electric potential $V$ :

$$
\nabla \cdot([\sigma+j \omega \varepsilon] \nabla V)=0 .
$$

The model consists of a simplified geometry, as shown in figure 6. Three pairs of source (injection) electrodes are placed on the surface of the thorax (each pair in one vertical line) and inject an alternating current with a magnitude of 5 $\mathrm{mA}$ and a frequency of $100 \mathrm{kHz}$ asynchronously. For each injection, the electric potential drop is evaluated between five measurement electrode pairs (each pair in one vertical line) which leads to the thoracic impedance

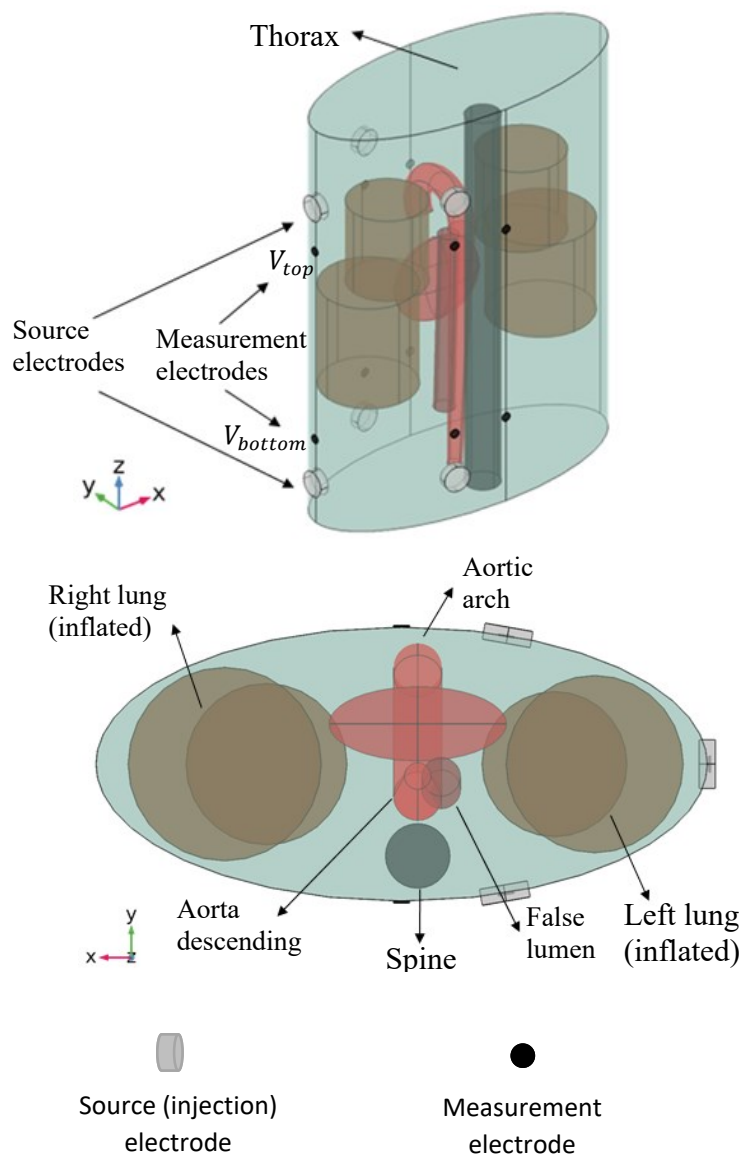

Fig. 6: Simulation model setup. a) 3D view - b) 2D bottom view.

$$
\underline{Z}=\frac{\underline{V}_{t o p}-\underline{V}_{\text {bottom }}}{\underline{I}}
$$

The boundary conditions are:

- $V=$ constant, on the top source electrode;

- $\int_{S}[\sigma+j \omega \varepsilon] \nabla V \cdot \mathrm{n} d S=I_{0}$, on the top source electrode;

- $V=0$, on the bottom source electrode;

- $\boldsymbol{n} .[\sigma+j \omega \varepsilon] \nabla V=0$, on the thorax surface

where $\boldsymbol{n}$ is the normal unit vector. A tetra-polar spot electrode configuration is used as proposed in [18]. Different positions for source and measurement electrodes have been chosen to reach the most noticeable discrepancy between the impedance cardiograms of the healthy and dissected conditions [7]. The conductivity and the permittivity of tissue types, which are considered in the simulation model, have been taken from data provided in [19]. For other surrounding materials such as muscles, fat, and ribs, which are not considered directly in the simulation model, a mean conductivity and permittivity is assigned to the thorax domain to provide a realistic value for the static thoracic impedance $Z_{0}$ of about $25 \Omega$, as reported in [8]. In order to reduce the computational cost, only the first half of the cardiac cycle is considered in the simulation model.

\section{Modelling physiological changes in the presence of the false} lumen

It has been shown in the literature $[21,22]$ that the blood flow is highly disturbed locally inside the aorta and changes to turbulent flow with strong recirculation. As depicted in figure 7, flow disturbances occur around the dissection, which inhibits the deformation and orientation of the RBCs. Thus, the flow shear rate and, consequently, the electrical properties of blood are altered. At the highest blood flow velocity and consequently the highest deformation and orientation rate of the RBCs, a remarkable difference in the electrical conductivity between the healthy (non-disturbed flow) and the aortic dissection conditions can be expected [6]. Since no experimental or simulation data exist regarding conductivity changes of blood in this kind of disturbed flow, it is assumed that with a radially growing false lumen also the blood flow disturbances increase and the conductivity changes decrease. To quantify this assumption, a damage factor $D F$ has been introduced, which is the ratio of the volume of the dissection to the maximum volume of the false lumen:

$$
D F=\frac{\pi \cdot R_{F L}^{2} \cdot h_{F L}}{\pi \cdot \max \left(R_{F L}^{2}\right) \cdot h_{F L}}=\frac{R_{F L}^{2}}{\max \left(R_{F L}^{2}\right)}
$$

where $R_{F L}$ and $h_{F L}$ are the radius and the height of the false lumen, respectively. The maximum value for the radius of the false lumen in the simulation model is $1.5 \mathrm{~cm}$. At this level, it is assumed that the flow disturbances in the descending aorta are at maximum (figure 8). 


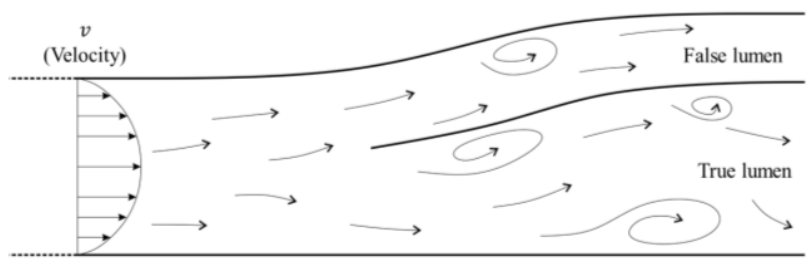

Fig.7: Flow disturbances around the dissection in case of an aortic dissection.

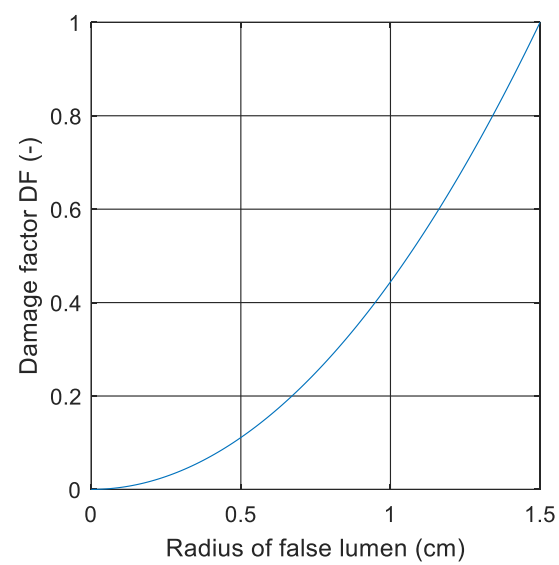

Fig.8: Damage factor DF as a function of the radius of the false lumen.

The damage factor $D F$ is applied to the conductivity changes of blood in the descending aorta during the cardiac cycle to model the decrease in the conductivity changes of blood due to dissection (see the section on Implementation of global sensitivity analysis ..., on the next page).

\section{Sensitivity analysis}

The aim of a Global Sensitivity Analysis (GSA) is to quantify the connection between the variance of the model output given the variability of its input. GSA is distinguished from a local sensitivity analysis since it investigates the whole input space of each random variable. This study aims to use a GSA technique to identify which electrode configuration has significant changes in the impedance cardiogram $-|d \underline{Z} / d t|$ given by the uncertainty on the developed status of $A D$. The discrepancies among obtained impedance cardiograms of the healthy and dissected models determine different states of the patient and are the criteria to simulate $A D$ identification.

One of the most known techniques in GSA is the variancebased method. Here, the output variance is portioned in the sum of the contributions of each random variable. Consider a mathematical model output $y$ as a function of an input random vector $\boldsymbol{x}$ of dimension $n$, the Sobol' decomposition reads as [22]

$$
\begin{aligned}
y=f\left(x_{1}, \ldots, x_{n}\right) & =f_{0}+\sum_{i=1}^{n} f_{i}\left(x_{i}\right) \\
& +\sum_{\substack{1 \leq i<j \leq n \\
f_{1, \ldots, n}}} f_{i j}\left(x_{i}, \ldots, x_{n}\right)+\cdots \\
&
\end{aligned}
$$

where $f_{0}$ is a constant. The integral of each summand of (5) is equal to zero, which ensures the uniqueness of the decomposition given the integrity of the model over the random input space, i.e. $\int f\left(x_{i}\right) f\left(x_{j}\right) d x_{i} d x_{j}=0$. The terms of (5) can be then derived univocally from the conditional expectation $E$ of the model output [23]

$$
\begin{gathered}
f_{0}=E[y] \\
f_{i}=E\left[y \mid x_{i}\right]-E[y] \\
f_{i j}=E\left[y \mid x_{i}, x_{j}\right]-f_{i}-f_{j}-E[y]
\end{gathered}
$$

A random variable $x_{i}$ is considered to be influential (noninfluential) to the model output if the conditional variance $V\left(E\left[y \mid x_{i}\right]\right)$ is large (small) enough compared to the variance of the quantity of interest. Therefore, the first-order sensitivity index (or main Sobol' index) can be derived as:

$$
S_{i}=\frac{V\left(E\left[y \mid x_{i}\right]\right)}{V(y)}
$$

which represents the contribution of the random variable $x_{i}$ to the change of model output, without taking into consideration the effect of its interaction with other input variables. Consequently, the total-order sensitivity index (or total Sobol' index), which evaluates the total effect of such input parameter, has to account for the conditional variance of the output, but conditioning to all factors except one, $\boldsymbol{x}_{\sim i}$ :

$$
\begin{aligned}
& S_{T i}=\frac{E\left[V\left(y \mid \boldsymbol{x}_{\sim i}\right)\right]}{V(y)} \\
&=1-\frac{V\left(E\left[y \mid \boldsymbol{x}_{\sim i}\right]\right)}{V(y)} .
\end{aligned}
$$

In this application, only the first-order and the total-order indices are considered. Any interaction between the input random variable can be derived by subtracting the first index to the total. Consequently, the difference will result in the amount of interaction present in the model.

The Sobol' indices are computed from a Polynomial Chaos Expansion (PCE) of the model [24], which also represents a valid mathematical metamodel. PCE consists of the sum of orthogonal, multivariate polynomials $\psi_{\alpha}$ of increasing order up to some maximal polynomial order $p$. The polynomials are multiplied by expansion coefficients $y_{\alpha}$, which can be estimated with different methods [25]. A PCE is written as:

$$
y(\boldsymbol{x}) \approx f_{P C E}(\boldsymbol{x})=\sum_{\alpha \in A} y_{\alpha} \psi_{\alpha}(\boldsymbol{x})
$$

where $\alpha$ is a multi-index that refers to the degree of each polynomial and each input parameter, the multivariate polynomials $\psi_{\alpha}$ are defined as the product of univariate polynomials of order $\alpha_{i}$, i.e. $\psi_{\alpha_{i}}$. The univariate polynomials are generated following the Askey scheme for the 
composition of polynomials [26]. Finally, from the PCE, it is possible to estimate the two sensitivity indices as the ratio between the PCE coefficients. Since the case study is evaluated in time, the implementation of time-dependent indices is implemented following [27] as:

$$
\begin{gathered}
S_{i} \approx \frac{\sum_{\alpha \in A_{i}} y_{\alpha}^{2}(t)}{\sum_{\alpha \in A ; \alpha \neq 1} y_{\alpha}^{2}(t)} \\
=\frac{\int_{0}^{t} \sum_{\alpha \in A_{i}} y_{\alpha}^{2}\left(t^{\prime}\right) d t^{\prime}}{\int_{0}^{t} \sum_{\alpha \in A ; \alpha \neq 1} y_{\alpha}^{2}\left(t^{\prime}\right) d t^{\prime}} \\
S_{T i} \approx \frac{\sum_{\alpha \in A_{i}^{T}} y_{\alpha}^{2}(t)}{\sum_{\alpha \in A ; \alpha \neq 1} y_{\alpha}^{2}(t)} \\
=\frac{\int_{0}^{t} \sum_{\alpha \in A_{i}^{T}} y_{\alpha}^{2}\left(t^{\prime}\right) d t^{\prime}}{\int_{0}^{t} \sum_{\alpha \in A ; \alpha \neq 1} y_{\alpha}^{2}\left(t^{\prime}\right) d t^{\prime}} .
\end{gathered}
$$

The PCE is computed through the UQLab toolbox for Matlab $[28,29]$. However, the time-dependent indices are developed manually from the extrapolation of the PCE coefficients.

\section{Implementation of global sensitivity analysis (GSA) to the} simulation model

To assess the sensitivity analysis, the input and output spaces together with the numerical models, have to be established. Since the aim of the study is to catch the difference between different health conditions, two numerical models are set. The first one refers to the healthy condition and the second one to the dissected condition. As described in section 2-2, the latter differs from the first one in the presence of the false lumen and another blood flow profile. Therefore, different input spaces are produced for each model. Besides, introducing variability in the input space of the models will guarantee the realization of as many patient-specific cases as the dimension of the input sample. Thus, a deeper understanding of the impedance cardiography for a human thorax can be revealed. From the models' evaluations, the PCEs for the healthy and dissected conditions are constructed, and analyzing the differences between them will guide toward the choice of the best electrode configuration.

The input space of the healthy case is composed of only two random variables, namely the maximum radius of the true lumen $R_{T L}$ and the blood conductivity coefficient $\theta_{H}$. $R_{T L}$ is considered uniformly distributed between 1.35 and $1.95 \mathrm{~cm}$, according to the study [30]. As it was shown in figure 2 , the average radius of the aorta changes in time due to pressure changes over a cardiac cycle. Since the aorta in the simulation model is considered as a blood-filled lumen, different values of the $R_{T L}$ emulates different blood volumes dilating the aorta, in other words, different stroke volume values. It has to be mentioned that changes in the stroke volume will also vary the peak velocity of the blood passing the aorta. Since for higher velocities, almost all the RBCs are entirely aligned and deformed, the differences among the blood conductivity changes for different stroke volumes are not significant. Therefore, here, for simplicity, it is neglected. Based on the distribution's moments of $R_{T L}$, the stroke volume changes approximately between 62 and $140 \mathrm{ml}$ in the simulation model.

The blood conductivity changes as a function of the reduced average velocity $\langle v / R\rangle$ for five different haematocrit levels are shown in figure 5 . For the parameterization of the haematocrit-dependent blood conductivity changes corresponding to other haematocrit values, the coefficient $\theta_{H}$ has been introduced. It emulates a scaling factor for $\Delta \sigma$ at a haematocrit level of $35 \%$ up to $55 \%$ at maximum. Given $h$ as the index that represents different haematocrit levels, the parameter $\theta_{H, h}$ is set as:

$$
\theta_{H, h}= \begin{cases}1 & \text { if } h=1 \\ 1+a_{h} & \text { if } h=2, \ldots, 5\end{cases}
$$

where $h=1$ to $h=5$ refer to $H=35 \%, H=40 \%$, $H=45 \%, H=50 \%$ and $H=55 \%$ respectively.

$$
a_{h}=\sum_{k=2}^{h}\left(\Delta \sigma_{H, k}-\Delta \sigma_{H, k-1}\right) / \Delta \sigma_{H, k-1} \text { computes the }
$$
difference in conductivity changes between two successive haematocrit levels, $\Delta \sigma_{H, k}$ are the conductivity changes of the blood for a given haematocrit level $H$ and index $k$. In conclusion, since the variable $H$ is uniformly distributed between $35 \%$ and $55 \%$, the parameter $\theta_{H, h}$ is also uniformly distributed between 1 and 1.1. Therefore, the blood conductivity changes for the healthy case are computed as:

$$
\Delta \sigma(v(t), H)=\left.\theta_{H, h} \cdot \Delta \sigma(v(t))\right|_{H=35 \%}
$$

where $v(t)$ is the velocity of blood and $\left.\Delta \sigma(v(t))\right|_{H=35 \%}$ represents the velocity-based conductivity changes for $H=35 \%$, as shown in figure 5 .

The dissected condition includes both the random variables of the healthy condition plus the radius of the false lumen $R_{F L}$ and the radial position of the false lumen to the true lumen $\alpha_{F L}$ (representing different possible positions of the false lumen). The two new parameters have been considered uniformly distributed since knowledge regarding the dimension and position of the false lumen is not available prior to the measurement, and the uniform distribution better represents the lack of knowledge regarding a model variable. The description of the input space for both case studies is given in table 1 . Furthermore, since in the case of aortic dissection the damage factor $D F$ also affects the conductivity changes, the blood conductivity results in:

$$
\begin{aligned}
& \sigma(v(t), H)= \sigma_{0}(H)+ \\
& \Delta \sigma(v(t), H) \cdot(1-D F)
\end{aligned}
$$


where $\sigma_{0}(H)$ is the initial conductivity of blood for a given haematocrit level. In equation (16), $D F=0$ corresponds to the conductivity of the flowing blood in the aorta for the healthy condition.

Table.1: Input space description for the healthy and dissected study cases.

\begin{tabular}{|c|c|c|c|c|}
\hline Cases & Variable & Distribution & Moments & Unit \\
\hline \multirow{3}{*}{ Healthy } & $R_{T L}$ & Uniform & {$[1.351 .95]$} & $\mathrm{cm}$ \\
\cline { 2 - 5 } & $\theta_{H}$ & Uniform & {$[1.01 .1]$} & - \\
\hline \multirow{4}{*}{ Dissected } & $R_{T L}$ & Uniform & {$[1.351 .95]$} & $\mathrm{cm}$ \\
\cline { 2 - 5 } & $\theta_{H}$ & Uniform & {$[1.01 .1]$} & - \\
\cline { 2 - 5 } & $R_{F L}$ & Uniform & {$[0.31 .5]$} & $\mathrm{cm}$ \\
\cline { 2 - 5 } & $\alpha_{F L}$ & Uniform & {$[2.93 .65]$} & $\mathrm{rad}$ \\
\hline
\end{tabular}

The two models produce a measurement of the impedance cardiograms for each source electrode pairs $N_{i n j}$ and for each measuring electrode pairs $N_{\text {meas }}$ at each time step $N_{t}$, as described earlier. The time interval is limited to the first half of the cardiac cycle. After setting the input and output space characteristics, the next step is to set up the options for the PCE. It is essential to notice that a minimum number of point evaluations $N_{S}$ is needed to have an accurate expansion. $N_{S}$ is defined to satisfy

$$
N_{s} \geq 2 \cdot \frac{(M+p) !}{(M ! p !)}
$$

where $M$ is the input space dimension, and $p$ is the order of the polynomial. The healthy condition model has $N_{s}$ equal to 100 , while for the dissected condition $N_{s}$ is assumed 150 . To ensure proper construction of the metamodel and an accurate representation of the interaction between the random input variables, polynomial order of 4 has been chosen for both models. Such a degree guarantees the presence of enough interaction terms in the expansion.

Two PCE functions $\widehat{H C}^{P C E}(t)$ and $\widehat{D C}^{P C E}(t)$ are introduced. They express the impedance cardiograms of the healthy condition $(H C)$ and the dissected condition $(D C)$, respectively:

$$
\begin{aligned}
& \widehat{H C}_{n, m}^{P C E}(t)=-\left|\frac{d \underline{Z}}{d t}\right|_{H C} \\
& \widehat{D C}{ }_{n, m}^{P C E}(t)=-\left|\frac{d \underline{Z}}{d t}\right|_{D C}
\end{aligned}
$$

where $n$ and $m$ are the indices related to the source and the measurement electrode pairs, respectively. The difference $\hat{Y}_{n, m}^{P C E}(t)$ between (22) and (23) is then calculated as (24)

$$
\widehat{Y}_{n, m}^{P C E}(t)=\widehat{H C}_{n, m}^{P C E}(t)-\widehat{D C}_{n, m}^{P C E}(t)
$$

which expresses the PCE output of the difference between the healthy and dissected metamodels for each electrode configuration and time step.

\section{Informed consent}

Informed consent has been obtained from all individuals included in this study.

\section{Ethical approval}

The conducted research is not related to either human or animal use.

\section{Results and discussion}

Three source electrode pairs (A, B, and $C$ ) and five measurement electrode pairs $\left(m_{1}\right.$ to $\left.m_{5}\right)$ are considered in the simulation models of the healthy and dissected conditions (figure 9).
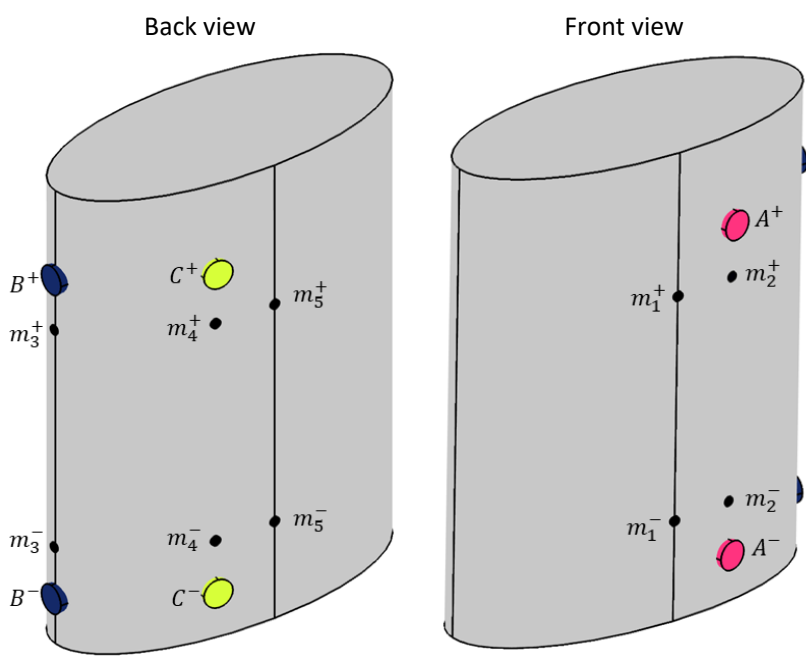

Fig.9: Source electrode pairs and measurement electrode pairs positions.

Each simulation contains an injection from one of the source electrode pairs and measuring from all the five measurement electrode pairs. For each simulation, the impedance cardiogram $-|d \underline{Z} / d t|$ is computed and through all the simulation results (for different input variables), two metamodels, $\widehat{H C}_{n, m}^{P C E}(t)$ and $\widehat{D C}_{n, m}^{P C E}(t)$ are evaluated. The objective is to find the setup which gives the maximum difference between the impedance cardiograms measured for the healthy and dissected conditions $\hat{Y}_{\max }^{P C E}$.

Combinations of injections have been applied, and the results of calculating $\hat{Y}_{n, m}^{P C E}(t)$ are shown in figure 10 . For each electrode configuration, the highest difference occurs in the fourth time step where, indeed, the blood velocity is at the peak level or in other words, the blood conductivity is the highest. It must be mentioned that in the classical ICG application the maximum value of the impedance cardiogram $-|d \underline{Z} / d t|_{\max }$ is used for calculating Stroke Volume (SV) and Cardiac Output (CO) [31]. 

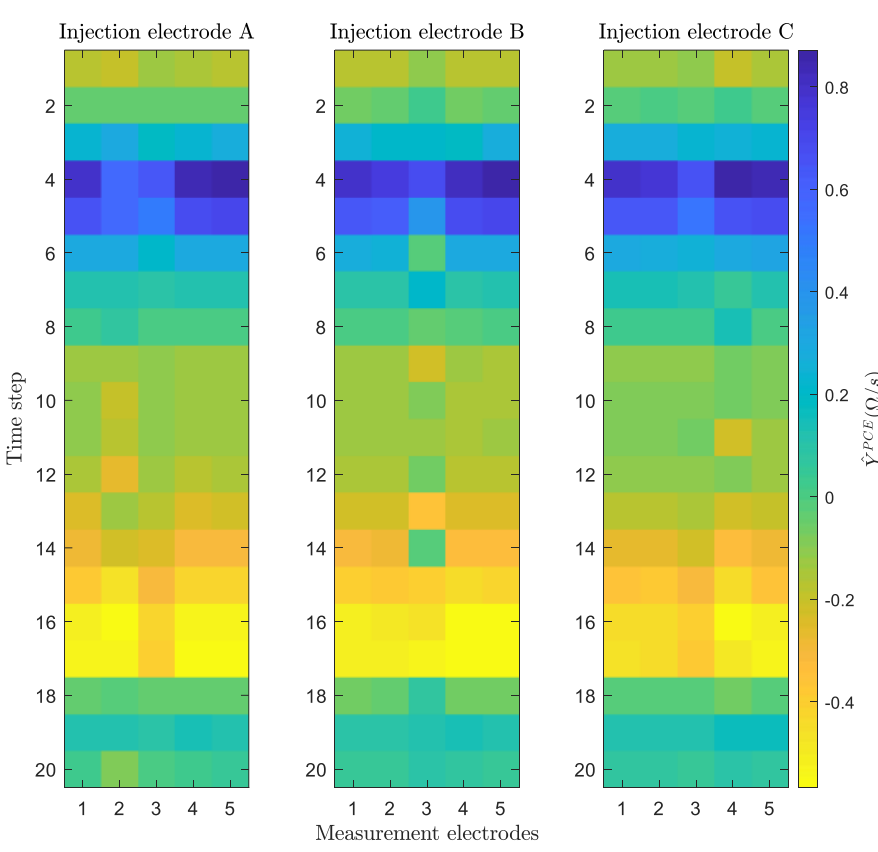

Fig.10: Values of $\widehat{Y}_{n, m}^{P C E}(t)$ reflecting the discrepancy between the healthy and dissected conditions for 20-time steps and all proposed electrode combinations.

$$
\hat{Y}_{\max }^{P C E}(\Omega / s)
$$

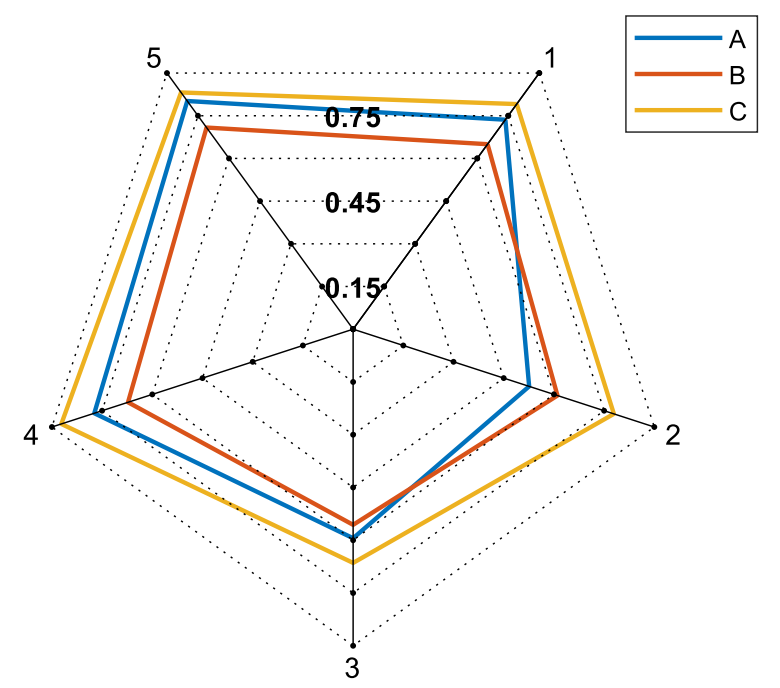

Fig.11: Maximal discrepancy $\hat{Y}_{\max }^{P C E}$ for the fourth time step and each electrode configuration. Colours show source electrodes; blue: injection from $A$, red: injection from $B$, yellow: injection from $C$; numbers show the measurement electrodes.

Thus, the $\hat{Y}_{\max }^{P C E}$ between the healthy and dissected conditions for all possible configurations has been plotted in figure 11. The best configuration for the diagnosis purpose is the one with the highest $\hat{Y}_{\max }^{P C E}$ value which is here reached by injecting from source electrodes $C$ and measuring from measurement electrode 4. Furthermore, false lumen expansion or false lumen thrombosis change the blood flow significantly inside both the true lumen and the false lumen. Thus, considering the highest $\hat{Y}_{\max }^{P C E}$ value makes sense, for tracking the status of the disease. Hence, injecting the electric current from the source electrodes $C$ (i.e. on the backside of the thorax) provides a more reliable outcome.

In figure 12 , the results of the sensitivity analysis on $\widehat{H C}_{C, 4}^{P C E}(t), \widehat{D C}_{C, 4}^{P C E}(t), \widehat{Y}_{C, 4}^{P C E}(t)$ for the most appropriate electrode setup (source electrodes $C$ and measurement electrode 4) is summarized. The first or main Sobol' index is a measure of the influence of a random variable to the output defined in (17). It can be observed from figure 12.a that, for the healthy case, the maximum radius of the true lumen $R_{T L}$ which emulates the stroke volume has the highest sensitivity while the sensitivity of the haematocrit on the blood conductivity changes $\left(\theta_{H}\right)$ is very low.

From the sensitivity analysis in the dissected case in figure 12.b, $R_{T L}$ is less sensitive than in the healthy case. The next most sensitive parameter is $R_{F L}$, which emulates the blood conductivity changes due to the existence of the false lumen. The sensitivity analysis of the difference between the healthy and the dissected cases $\hat{Y}_{C, 4}^{P C E}(t)$ (figure 12.c) shows that $R_{F L}$ has the highest sensitivity thus the highest impact on the output $\left(\hat{Y}_{C, 4}^{P C E}(t)\right)$ followed by $R_{T L}$ while $\theta_{H}$ and $\alpha_{F L}$ are not sensitive at all. Furthermore, $R_{F L}$ has a shallow interaction rate with the other random variables, which can be seen in the highest value of the total Sobol index defined in (18). This low correlation is a significant feature since interactions could affect the final result in a non-predictable way. Thus, as expected, the discrepancy between the impedance cardiograms of the healthy and dissected cases mostly originates from the random variable $R_{F L}$ and consequently, the damage factor $D F$.

In figure $13, \hat{Y}_{\max }^{P C E}$ is shown as a function of the damage factor $D F$ for the injection from source electrodes $C$.

$\hat{Y}_{\max }^{P C E}$ increases almost linearly with the damage factor, which means that the probability to identify an aortic dissection grows as the disease progresses. This issue is depicted in figure 14.a by showing $\widehat{H C}_{C, 4}^{P C E}(t)$ and $\widehat{D C}_{C, 4}^{P C E}(t)$ for different values of the damage factor $D F$ and in figure 14.b by showing the difference between them $\hat{Y}_{C, 4}^{P C E}(t)$. On the other hand, these changes are also observable in the evaluation of the Stroke Volume (SV) and the Cardiac Output (CO), which leads to distorted values.

To summarize, in the first stages of the $A D$ in which the existence of the false lumen does not make apparent changes to the rheology of the blood flow, the presence of the disease by impedance cardiography might not be noticeable. However, as soon as the dissection creates remarkable pathological changes in the cardiovascular system, the changes in the measured impedance cardiogram due to the development of the disease such as false lumen expansion and false lumen thrombosis, might be trackable. 

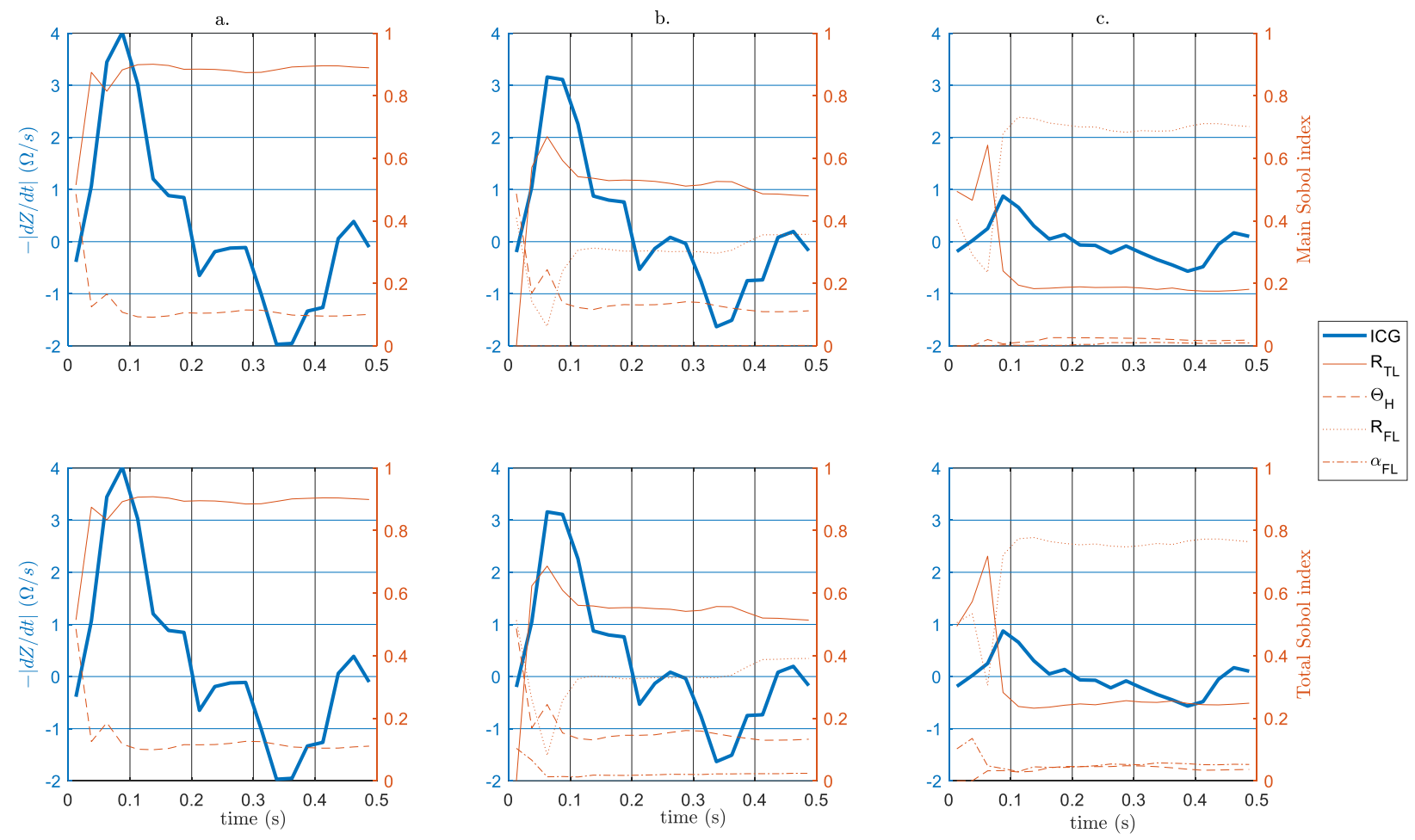

Fig.12: Sensitivity analysis on a. $\widehat{H C}_{C, 4}^{P C E}(t)$, b. $\widehat{D C}_{C, 4}^{P C E}(t)$, c. $\hat{Y}_{C, 4}^{P C E}(t)$.

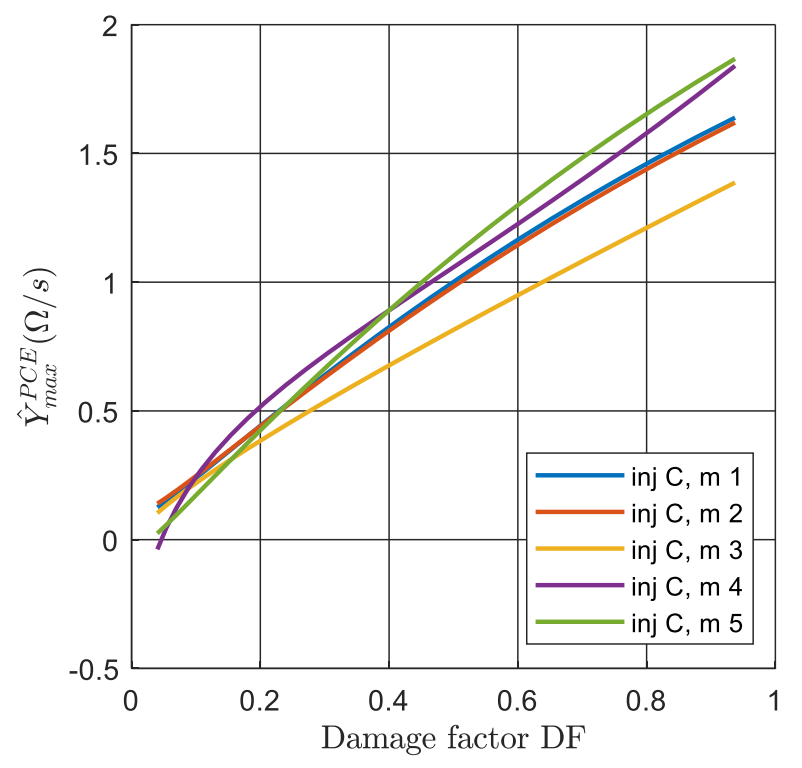

Fig.13: Changing of $\hat{Y}_{\max }^{P C E}$ by the damage factor for injection from source electrodes $\mathrm{C}$ (inj $\mathrm{C}$ ) and measurement from five electrode pairs ( $\mathrm{m} 1$ to $\mathrm{m} 5$ ).

\section{Conclusion}

This study aims to investigate different electrodes configurations concerning the discrepancy between the healthy case and type $B$ aortic dissection case. For this purpose, a numerical simulation model using a simplified geometry of the thorax has been set up. Since there are many uncertainties regarding the parameters that affect the results, a Global Sensitivity Analysis (GSA) has been implemented to quantify the relation of the variance of the model output (impedance cardiogram $-|d \underline{Z} / d t|$ ) and the variability of its input. The sensitivity analysis of the models' output shows that the highest difference between the impedance cardiograms of the healthy and dissected conditions occurs when the velocity is highest in the aorta. Also, the highest difference in the maximum value of the impedance cardiograms of the healthy and dissected conditions $\hat{Y}_{\text {max }}^{P C E}$, can be gained when the source and measurement electrode pairs are positioned on the back of the thorax.

It has been shown that the size of the false lumen has a tremendous effect on the impedance cardiogram of the dissected condition. This effect shows that in some cases, the pathological changes caused by false lumen might end up in different calculated haemodynamic parameters by impedance cardiography in comparison to other methods. Furthermore, the development of the aortic dissection disease such as false lumen expansion and false lumen thrombosis cause some pathological changes which will alter the measured impedance cardiogram. Thus, applying impedance cardiography to track these changes can be helpful for the medical management of AD.

For future works, the electrical conductivity changes of the blood in case of disturbed aortic flow by setting up simulation models and experiments, and also the possibility of tracking false lumen thrombosis by impedance cardiography, will be investigated. 

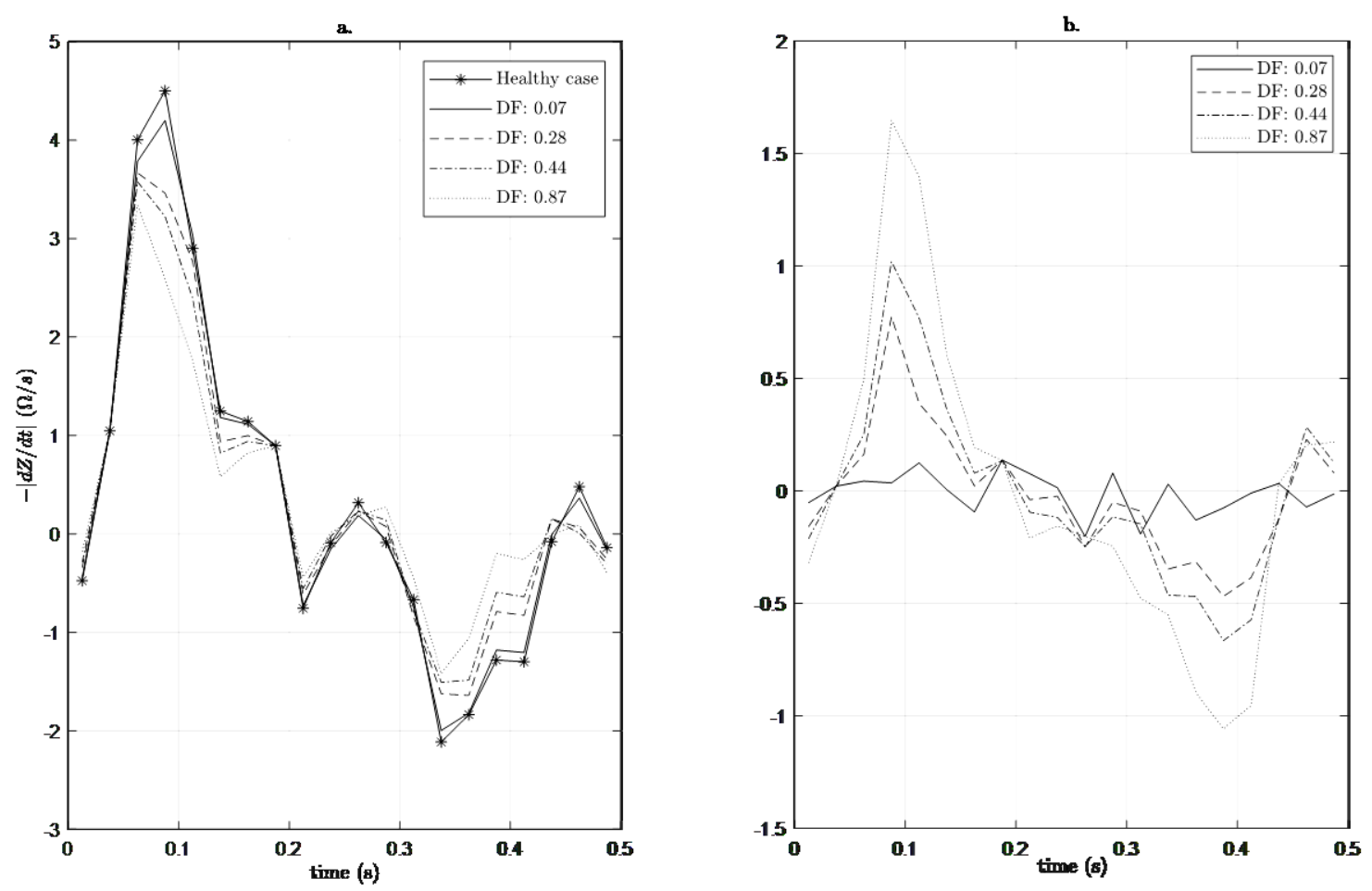

Fig.14: a. $\widehat{H C}_{C, 4}^{P C E}(t)$ and $\widehat{D C}_{C, 4}^{P C E}(t)$ for different damage factors, b. $\widehat{Y}_{C, 4}^{P C E}$ for different damage factors.

\section{Acknowledgement}

This work is supported by Graz University of Technology through the LEAD Project "Mechanics, Modelling, and Simulation of Aortic Dissection". Also, the authors would like to acknowledge the use of high power computing resources provided by the ZID of Graz University of Technology.

\section{Conflict of interest}

Authors state no conflict of interest.

\section{References}

1. Khan IA, Nair CK. Clinical, diagnostic and management perspectives of aortic dissection. Elsevier Chest. 2002; 122(1): 311-28. https://doi.org/10.1378/chest.122.1.311

2. Heuser J. Distributed under a CC-BY-SA-3.0 license Wikimedia Commons. 2016.

3. Patchett N. Distributed under a CC BY-SA 4.0 license. Wikimedia Commons. 2015.

4. Altamirano-Diaz L, Welisch E, Dempsey AA, Park TS, Grattan $M$, Norozi K. Non-invasive measurement of cardiac output in children with repaired coarctation of the aorta using electrical cardiometry compared to transthoracic Doppler echocardiography. Physiol Meas. 2018; 17;39(5): 055003. https://doi.org/10.1088/1361-6579/aac02b

5. Reinbacher-Köstinger A, Badeli V, Biro O, Magele C. Numerical simulation of conductivity changes in the human thorax caused by aortic dissection. IEEE Trans. Magnetic. 2019;55(6): 5100304. https://doi.org/10.1109/tmag.2019.2895418

6. Badeli V, Reinbacher-Köstinger A, Biro O, Magele C. Numerical simulation of impedance cardiogram changes in case of chronic aortic dissection. Springer, Singapore. 2020;
In: Bertemes-Filho P. (eds) 17th International Conference on Electrical Bioimpedance. ICEBI 2019. IFMBE Proceedings, vol 72. https://doi.org/10.1007/978-981-13-3498-6_9

7. Reinbacher-Köstinger A, Badeli V, Melito GM, Magele C, Biro $O$. Numerical simulation of various electrode configurations in impedance cardiography to identify aortic dissection. Springer, Singapore. 2020; In: Bertemes-Filho P. (eds) 17th International Conference on Electrical Bioimpedance. ICEBI 2019. IFMBE Proceedings, vol 72. https://doi.org/10.1007/978-981-13-3498-6_7

8. Bernstein DP. Impedance cardiography: Pulsatile blood flow and the biophysical and electrodynamic basis for the stroke volume equations. J Electr Bioimp. 2010; 1: 2-17. https://doi.org/10.5617/jeb.51

9. Ulbrich M, Muhlsteff J, Leonhardt S, Walter M. Influence of physiological sources on the impedance cardiogram analyzed using 4D FEM simulations. Physiol. Meas. 2014; 35: 14511468. https://doi.org/10.1088/0967-3334/35/7/1451

10. de Sitter A, Verdaasdonk RM, Faes TJC. Do mathematical model studies settle the controversy on the origin of cardiac synchronous trans-thoracic electrical impedance variations? A systematic review. Physiol. Meas. 2016; 37: R88-R108. https://doi.org/10.1088/0967-3334/37/9/r88

11. Alastruey J, Xiao N, Fok H, Schaeffter T, Figueroa CA. On the impact of modelling assumptions in multi-scale, subjectspecific models of aortic haemodynamics. J. Roy. Soc. Interface. 2016; 13(119): 20160073. https://doi.org/10.1098/rsif.2016.0073

12. Visser KR. Electric properties of flowing blood and impedance cardiography. Ann. Biomed. Eng. 1989; 17: 463-473. https://doi.org/10.1007/bf02368066 
13. Hoetink AE, Faes TJ, Visser KR, Heethaar RM. On the flow dependency of the electrical conductivity of blood. IEEE Trans. Biomed. Eng. 2004; 51(7): 1251-1261. https://doi.org/10.1109/tbme.2004.827263

14. Fuji M, Nakajima K, Sakamoto K, Kanai H. Orientation and deformation of erythrocytes in flowing blood. Annals of the New York Academy of Sciences. 1999; 873(1): 245-61. https://doi.org/10.1111/j.1749-6632.1999.tb09473.x

15. Gaw RL, Cornish BH, Thomas BJ. The electrical impedance of pulsatile blood flowing through rigid tubes: an experimental investigation. 13th International Conference on Electrical Bioimpedance and the 8th Conference on Electrical Impedance Tomography. 2007; pp. 73-76. https://doi.org/10.1007/978-3-540-73841-1_22

16. Gaw RL, Cornish BH, Thomas BJ. The electrical impedance of pulsatile blood flowing through rigid tubes: $A$ theoretical investigation. IEEE Transaction on Biomedical Engineering. 2008; 55(2): 721-727. https://doi.org/10.1109/tbme.2007.903531

17. COMSOL Multiphysics. v. 5.3. COMSOL AB, Stockholm, Sweden.

18. Mansouri S, Alhadidi T, Chabchoub S, Salah RB. Impedance cardiography: Recent applications and developments. Biomedical Research. 2018; 29 (19): 3542-3552. https://doi.org/10.4066/biomedicalresearch.29-17-3479

19. Gabriel S. The dielectric properties of biological tissues. Physics in Medicine and Biology. 1996; 41: 2231-2249.

20. Chen $D$, Müller-Eschner $M$, von Tengg-Kobligk $H$, Barber $D$, Böckler D, Hose R, Ventikos Y. A patient-specific study of Type-B aortic dissection: evaluation of true-false lumen blood exchange. BioMedical Engineering OnLine. 2012; 12: 65. https://doi.org/10.1186/1475-925x-12-65

21. Cheng Z, Tan FP, Riga CV, Bicknell CD, Hamady MS, Gibbs RG, Wood NB, Xu XY. Analysis of flow patterns in a patientspecific aortic dissection model. Journal of Biomechanical Engineering. 2010; 132(5), 051007. https://doi.org/10.1115/1.4000964
22. Sobol' IM. Sensitivity estimates for nonlinear mathematical models. Math Modeling Comput Exp. 1993; 1: 407-14.

23. Saltelli A. et al. Global sensitivity analysis: the primer. John Wiley \& Sons. 2008.

24. Sudret B. Global sensitivity analysis using polynomial chaos expansions. Reliability Engineering \& System Safety. 2008; 93(7): 964-979. https://doi.org/10.1016/j.ress.2007.04.002

25. Crestaux T, Maître OL, Martinez J-M. Polynomial chaos expansion for sensitivity analysis. Reliability Engineering \& System Safety. 2009; 94.7: 1161-1172. https://doi.org/10.1016/j.ress.2008.10.008

26. Xiu D, Karniadakis GE. The Wiener - Askey polynomial chaos for stochastic differential equations. SIAM Journal on Scientific Computing. 2002; 24.2: 619-644. https://doi.org/10.1137/s1064827501387826

27. Alexanderian A, Gremaud PA, Smith RC. Variance-based sensitivity analysis for time-dependent processes. Reliability Engineering \& System Safety. 2019; 106722. https://doi.org/10.1016/j.ress.2019.106722

28. Marelli S, Lamas C, Sudret B. UQLab user manual - Sensitivity analysis. Report UQLab-V1.3-106, Chair of Risk, Safety \& Uncertainty Quantification, ETH Zurich. 2019. https://doi.org/10.1061/9780784413609.257

29. Marelli S, Sudret B. UQLab user manual - Polynomial Chaos Expansions. Report UQLab-V1.3-104, Chair of Risk, Safety \& Uncertainty Quantification, ETH Zurich. 2019. https://doi.org/10.1061/9780784413609.257

30. Wolak $A$, et al. Aortic size assessment by noncontrast cardiac computed tomography: normal limits by age, gender, and body surface area. JACC: Cardiovascular Imaging. 2008; 1(2): 200-209. https://doi.org/10.1016/j.jcmg.2007.11.005

31. Bernstein DP, Lemmens HJM. Stroke volume equation for impedance cardiography. Medical \& Biological Engineering \& Computing. 2005; 43(4): 443-450. 